\title{
ENTROPY GENERATION ON MHD SLIP FLOW OVER A STRETCHING CYLINDER WITH HEAT GENERATION/ABSORPTION
}

\author{
S. JAIN* and S. BOHRA \\ Department of Mathematics and Statistics \\ Manipal University Jaipur \\ Jaipur-303007, INDIA \\ E-mail: shalini.jain@jaipur.manipal.edu
}

\begin{abstract}
In the present study, we have investigated entropy generation on a magnetohydrodynamic fluid flow and heat transfer over a stretching cylinder with a porous medium in slip flow regime. A uniform heat source and radiation is also considered. Similarity transformation has been applied for making an ordinary differential equation from nonlinear governing partial differential equations. The numerical solution for the set of nonlinear ordinary differential equations has been obtained by using the fourth-order Runge-Kutta scheme together with the shooting method. The effects of pertinent parameters such as the magnetic field parameter, permeability parameter, slip parameter, Prandtl number and radiation parameter on the fluid velocity distribution, temperature distribution, entropy generation and Bejan number are discussed graphically.
\end{abstract}

Key words: magnetohydrodynamic, heat generation, slip, entropy.

\section{Introduction}

The study of heat transfer around cylinders has received much attention in recent years due to its many important practical applications such as electronics cooling, thermal design of buildings, solar collectors, drilling operations, geothermal power generation, commercial refrigeration and float glass production, etc. A boundary layer viscous fluid flow and heat transfer characteristics outside a moving or stretching cylinder is an important problem in extrusion processes. The technological applications of such studies include fiber production and hot rolling. Lin and Shih [1,2] investigated laminar boundary layer heat transfer along horizontal and vertical moving cylinders with constant velocity. Wang [3] was first who studied the fluid flow and heat transfer characteristics outside the stretching hollow cylinder. For large Reynolds number, he compared numerical results with asymptotic solutions. The axisymmetric motion of a viscous fluid was studied by Burde [4] over a stretching cylinder. Further Ishak and Nazar [5] and Bachok and Ishak [6] investigated heat transfer characteristics of an axisymmetric laminar boundary layer flow outside a stretching cylinder with numerical solutions by converting governing equations into ordinary differential equations, which contain a curvature parameter. Earlier, the problem of laminar natural convection boundary layer flow over a vertical cylinder was studied by Sparrow and Gregg [7]. Chamkha and Quadri [8] studied heat and mass transfer in the presence of an internal heat source from a permeable cylinder immersed in a porous medium with a magnetic field. They applied heat source effect. Saeid [9] examined a free convective fluid flow and heat transfer from a horizontal cylinder by using a thermally nonequilibrium model. Nguyen et al. [10] and Gang et al. [11] examined the transient behavior of a viscous flow over an expanding stretching cylinder and a rotating circular cylinder. respectively.

Flow and heat transfer with slip has its applications in several engineering and industrial processes. Heat transfer can be enhanced by considering velocity slip. Due to this reason the fluid flow problems with slip have attracted attention of researchers. The laminar boundary layer flow due to a stretching cylinder in the presence of slip was studied by Wang and $\mathrm{Ng}$ [12] and Mukhopadhyay [13]. Chauhan et al. [14, 15]

\footnotetext{
* To whom correspondence should be addressed
} 
considered the slip flow and heat transfer through a porous medium outside a hollow stretching cylinder in the presence of a magnetic field. Recently, Jain and Parmar [16] examined the heat and mass transfer characteristics in an MHD flow through a stretching cylinder in the heat source.

The radiation effect has an important role in the context of space technology and in the processes involving high temperatures. Jain and Bohra $[17,18]$ studied fluid flow and heat transfer over a rotating disk and rotating channel through a porous medium with radiation effect and in different conditions. Abbas et al. [19] analyzed the radiation effect on a laminar MHD viscous fluid flow over a stretching cylinder in porous medium. Jain and Chaudhary $[20,21]$ investigated the effect of radiation on heat and mass transfer over a moving cylinder.

Heat generation/absorption has its application in problems dealing with dissociating fluids, concerned with chemical reactions, may alter the particle deposition rate. Abel et al. [22] gave on analytical solution for the problem of flow and heat transfer of an incompressible viscous fluid over a continuously moving permeable stretching surface with nonuniform heat source/sink in a fluid-saturated porous medium. The effects of heat source, chemical reaction and suction/injection over a permeable cylinder moving with a linear velocity were studied by Chamkha [23]. Manjunatha et al. [24] took a stretching cylinder to observe the effect of heat generation/absorption on the flow and heat transfer of an MHD dusty fluid, which is embedded in porous medium.

The analysis of entropy generation minimization in a thermal system was pioneered by Bejan [25]. Based on his idea, many researchers have examined entropy generation effects in flow and heat transfer systems such as Makinde and Beg [26] and Jain et al. [27, 28]. The minimization of entropy over an isothermal rotating cylinder was investigated by Bak and Heilen [29] and Yilbas [30]. Butt and Ali [31] discussed entropy generation effect on a magnetohydrodynamic flow and heat transfer over a radially stretching sheet. Recently, entropy production in a viscous flow over an oscillating stretching cylinder was examined by Munawar et al. [32]. Butt and Ali [33] contributed to the study of entropy generation effects in MHD fluid flow over a stretching cylinder. Motivated by the studies mentioned above, in the present study we have investigated entropy generation for a magnetohydrodynamic slip flow over a stretching cylinder in a porous medium with radiation and uniform heat source.

\section{Flow analysis}

Consider a steady, radiative, laminar, boundary layer flow of an electrically conducting viscous fluid driven by a stretching cylinder of diameter $2 \mathrm{a}$, in a fluid saturated porous medium along the axial of the cylinder and $r$ is measured in the radial direction and $\mathrm{z}$ is in axial direction. A uniform magnetic field $B_{0}$ is applied along the radial direction. An induced magnetic field has been neglected as the magnetic Reynolds number considered is small. Considering the assumptions mentioned and Chauhan et al. [14].

It can be written as

$$
\begin{aligned}
& \frac{\partial(r u)}{\partial r}+\frac{\partial(r w)}{\partial z}=0 \\
& u \frac{\partial u}{\partial r}+w \frac{\partial u}{\partial z}=-\frac{1}{\rho} \frac{\partial P}{\partial r}+v\left(\frac{\partial^{2} u}{\partial r^{2}}+\frac{1}{r} \frac{\partial u}{\partial r}-\frac{u}{r^{2}}\right)-\frac{v u}{k_{0}} \\
& u \frac{\partial w}{\partial r}+w \frac{\partial w}{\partial z}=v\left(\frac{\partial^{2} w}{\partial r^{2}}+\frac{1}{r} \frac{\partial w}{\partial r}\right)-\frac{\sigma B_{0}^{2} w}{\rho}-\frac{v w}{k_{0}}
\end{aligned}
$$

Subject to the boundary conditions 
at

$$
r=a: \quad u=0, \quad w=2 c z+\alpha^{*} \frac{\partial w}{\partial r}
$$

at

$$
r \rightarrow \infty: \quad w \rightarrow 0
$$

where, $u$ and $w$ are the velocity components in the $r$ and $z$ directions, respectively; $c>0$ is a positive constant; $\mu$ is viscosity; $\nu=\mu / \rho$ is the kinematic viscosity; $\rho$ is fluid density; $\sigma$ is the electrical conductivity; $k_{0}$ is the permeability of the porous medium; $P$ is pressure; $\alpha^{*}$ is the velocity slip parameter.

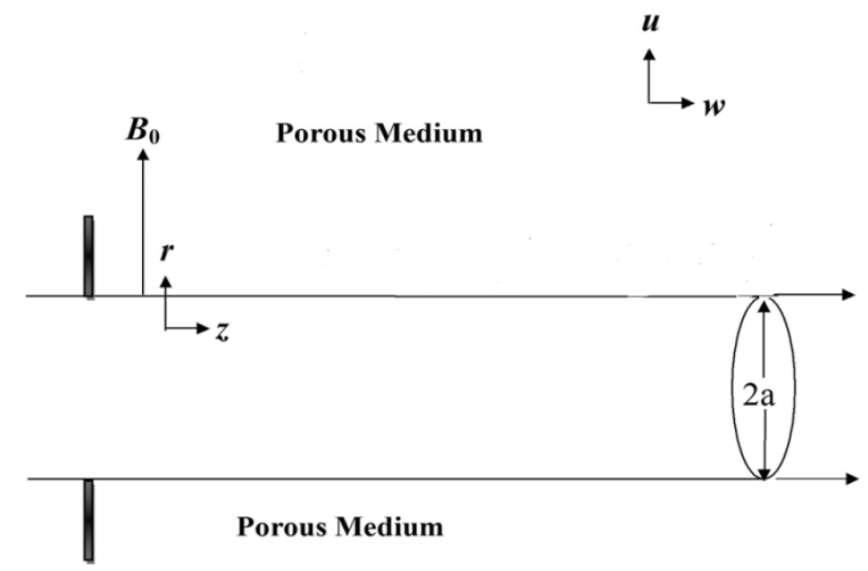

Fig.1. Schematic diagram of the problem.

We assume that $w_{e}(z)=2 c z$, so Eqs (2.1) to (2.3) admit similarity solutions.

Similarity transformations have been applied in the governing equations to reduce them into the corresponding ordinary differential equations, following Chauhan et al. [14] and Wang [3]

$$
\eta=\left(\frac{r}{a}\right)^{2}, \quad u=\frac{-c a f(\eta)}{\sqrt{\eta}}, \quad w=2 c z f^{\prime}(\eta)
$$

Substituting Eq.(2.5) into Eqs (2.1) - (2.4), Eq.(2.1) is identically satisfied and from Eq.(2.2), we obtain

$$
\eta f^{\prime \prime \prime}+f^{\prime \prime}+\operatorname{Re}\left(f f^{\prime \prime}-f^{\prime 2}\right)-\left(M^{2}+\frac{1}{K}\right) f^{\prime}=0
$$

where a prime denotes differentiation with respect to $\eta$ and $\operatorname{Re}=\frac{c a^{2}}{2 v}$ is the Reynolds number, gives the information, whether the flow is inertial or viscous force dominant and it describes about the flow whether it is laminar or turbulent. For a laminar flow the range of the Reynolds number is 2000. For transition flow the local Reynolds number values are between 2000 to 4000 and for values higher than 4000, the flow becomes turbulent. Here we study at low Reynolds number for laminar flow.

$M=\sqrt{\frac{\sigma B_{0}^{2} a^{2}}{4 v \rho}}$ is the magnetic parameter applied normal to the flow which creates a resistive type force, the Lorentz force and $K=\frac{k_{0}}{a^{2}}$ is permeability parameter.

The corresponding boundary conditions are reduced to the following. 
at

$$
\eta=1: \quad f=0, \quad f^{\prime}=1+\alpha f^{\prime \prime},
$$

at

$$
\eta \rightarrow \infty: \quad f^{\prime} \rightarrow 0
$$

where, $\alpha=2 \alpha^{*} / a$ is velocity slip parameter.

\section{Heat transfer analysis}

The energy equation for the slip flow in the presence of heat absorption/generation is given as

$$
\rho C_{p}\left(u \frac{\partial T}{\partial r}+w \frac{\partial T}{\partial z}\right)=\kappa\left(\frac{\partial^{2} T}{\partial r^{2}}+\frac{1}{r} \frac{\partial T}{\partial r}\right)+Q_{0}\left(T-T_{\infty}\right)-\frac{\partial q_{r}}{\partial r}+\mu\left(\frac{\partial w}{\partial r}\right)^{2}
$$

where $T$ is the fluid temperature and the surface of the stretching cylinder is held at constant temperature $T_{w}$. The ambient fluid temperature is considered to be $T_{\infty}$, with $T_{w}>T_{\infty}$. Further, it is assumed that the fluid and the porous medium are in local thermal equilibrium.

Subject to the boundary conditions

$$
\begin{array}{ll}
\text { at } & r=a: \quad T-T_{w}=\beta^{*} \frac{\partial T}{\partial r}, \\
\text { at } \quad r \rightarrow \infty: \quad T \rightarrow T_{\infty} .
\end{array}
$$

$C_{P}$ is the specific heat at constant pressure; $\kappa$ is the thermal conductivity in the porous medium and $\beta^{*}$ is the temperature slip parameter, $Q_{0}$ is the heat generation/absorption coefficient and $q_{r}$ is the radiative heat flux.

Using the Rosseland approximation for radiation, the radiative heat flux is defined as

$$
q_{r}=-\frac{4 \sigma_{1}}{3 k_{1}} \frac{\partial T^{4}}{\partial y}
$$

where $\sigma_{1}$ is the Stephan-Boltzmann constant and $k_{1}$ is the mass absorption coefficient. The temperature differences within the flow are assumed to be sufficiently small so that $T^{4} \cong 4 T_{\infty}^{3} T-3 T_{\infty}^{4}$,

$$
\frac{\partial q_{r}}{\partial r}=\frac{\partial}{\partial r}\left(\frac{-4 \sigma_{1}}{3 k_{1}} \frac{\partial T^{4}}{\partial r}\right)=\frac{\partial}{\partial r}\left(\frac{-4 \sigma_{1}}{3 k_{1}} \frac{\partial\left(4 T_{\infty}^{3} T-3 T_{\infty}^{4}\right)}{\partial r}\right)=\frac{-16 \sigma_{1} T_{\infty}^{3}}{3 k_{1}} \frac{\partial^{2} T}{\partial r^{2}}
$$

Similarity transformation is

$$
\theta(\eta)=\frac{T-T_{\infty}}{T_{w}-T_{\infty}}
$$

Substituting Eqs (3.3) and (3.4) into Eqs (3.1) and (3.2), we get 


$$
\theta^{\prime \prime} \eta\left(1+\frac{4 N}{3}\right)+\theta^{\prime}\left(1+\operatorname{Re} \operatorname{Pr} f+\frac{2 N}{3}\right)+\frac{\operatorname{Pr} \operatorname{Re} \lambda}{2} \theta+\operatorname{Ec} \operatorname{Pr} f^{\prime \prime 2} \eta=0
$$

where $\operatorname{Pr}=\frac{\mu C_{P}}{\kappa}$ is Prandtl number. The Prandtl number signifies the ratio of momentum diffusivity to thermal diffusivity. Increasing the value of the Prandtl number increases the rate of cooling in the fluid flow as for higher Prandtl number, heat can diffuse from the wall faster and thickness of the thermal boundary layer decreases. Gases typically have Prandtl numbers in the range 0.71 while the Prandtl number for most liquids is much larger than unity. The Prandtl number for water ranges from 4-7, while that for oil might be of the order of $50-100$.

$$
N=\frac{4 \sigma_{1} T_{\infty}^{3}}{k_{l} \kappa} \text { is the radiation parameter. It defines the relative contribution of conduction heat transfer }
$$

to the thermal radiation transfer. For $N=1$, both modes of heat transfer have the same contribution.

$N>1$, thermal radiation contributes more than thermal conduction and for $N<1$; thermal radiation dominates over thermal conduction.

$$
\lambda=\frac{Q_{0}}{c \rho C_{P}} \text { is the heat absorption/generation parameter, in case of heat generation, } \lambda>0
$$

significantly accelerates the flow and for heat absorption $\lambda<0$, the flow is retarded (momentum boundary layer thickness is lowered).

$\mathrm{Ec}=\frac{w_{e}^{2}(z)}{C_{p}\left(T_{w}-T_{\infty}\right)}$ is the local Eckert number. It defines as the ratio of kinetic energy at the wall to the specific enthalpy difference between wall and fluid.

Now the corresponding boundary conditions for $\theta(\eta)$ as

at

$$
\eta=1: \quad \theta=1+\beta \theta^{\prime},
$$

at

$$
\eta \rightarrow \infty: \quad \theta \rightarrow 0
$$

where $\beta=2 \beta^{*} / a$ is the temperature slip parameter.

\section{Entropy generation analysis}

Following Bejan [25] and Butt and Ali [33], the dimensional volumetric rate of entropy generation for the present problem in cylindrical coordinates can be written as

$$
S^{\prime \prime \prime}=\frac{\kappa}{T_{r}^{2}}\left[\left(\frac{\partial T}{\partial r}\right)^{2}+\frac{16 \sigma_{1} T_{\infty}^{3}}{3 \kappa k_{1}}\left(\frac{\partial T}{\partial r}\right)^{2}\right]+\frac{\mu}{T_{r}}\left[\frac{w^{2}}{k_{0}}+\left(\frac{\partial w}{\partial r}\right)^{2}\right]+\frac{\sigma B_{0}^{2} w^{2}}{T_{r}}
$$

where $T_{r}$ is the reference temperature.

Using Eq.(2.6), we express the entropy generation number in a dimensionless form as

$$
N s=\frac{S^{\prime \prime \prime}}{S_{0}^{\prime \prime \prime}}=\frac{1}{X}\left[\left(1+\frac{4 N}{3}\right) \eta \theta^{\prime 2}+\frac{\mathrm{Br}}{\Omega}\left(\frac{f^{\prime 2}}{4 K}+\eta f^{\prime \prime 2}\right)+\frac{\mathrm{Br}}{\Omega}\left(M^{2} f^{\prime 2}\right)\right]
$$


where $S_{0}^{\prime \prime \prime}=\frac{4 \kappa\left(T_{w}-T_{\infty}\right)^{2}}{T_{r}^{2} L^{2}}$ is the reference volumetric entropy generation, $X=\left(\frac{a}{L}\right)^{2}$ is the dimensionless parameter, $\Omega=\frac{T_{w}-T_{\infty}}{T_{r}}$ is the dimensionless reference temperature, and $\mathrm{Br}=\frac{\mu w_{e}^{2}(z)}{\kappa\left(T_{w}-T_{\infty}\right)}$ is the Brinkman number. It is the ratio between heat produced by viscous dissipation and heat transported by molecular conduction. i.e., the ratio of viscous heat generation to external heating. The Brinkman number (Br) specifies the temperature rise due to viscous dissipation in the fluid.

For most fluids (air, water) at moderate speeds, the Brinkman number is much less than one.

$$
N s=N_{H}+N_{f}+N_{m}
$$

where $N_{H}, N_{f}$ and $N_{m}$ are entropy generation due to heat transfer, fluid friction and magnetic field., which are given as

$$
N_{H}=\frac{1}{X}\left(1+\frac{4 N}{3}\right) \eta \theta^{\prime 2}, \quad N_{f}=\frac{1}{X}\left(\frac{\mathrm{Br}}{\Omega}\right)\left(\frac{f^{\prime 2}}{4 K}+\eta f^{\prime \prime 2}\right) \quad \text { and } \quad N_{m}=\frac{1}{X}\left(\frac{\mathrm{Br}}{\Omega}\right)\left(M^{2} f^{\prime 2}\right)
$$

In many engineering designs and optimization problems, the contribution of the heat transfer entropy generation to the total entropy generation rate is required; therefore, Paoletti et al. [34] presented an alternative irreversibility distribution parameter in terms of Bejan number $(\mathrm{Be})$ and defined it as the ratio of the entropy generation due to heat transfer $\left(N_{H}\right)$ to the total entropy generation $(N s)$. The Bejan number is given by the following mathematical expression

Equation

$$
\mathrm{Be}=\frac{N_{H}}{N S} .
$$

Analyzing the expression for the Bejan number, it could be observed that its value lies between $0<\mathrm{Be}<1$ with the following extreme cases and when entropy is generated by heat transfer irreversibility and fluid friction irreversibility, the value of the Bejan number could be $\mathrm{Be}=1$ and $\mathrm{Be}=0$, respectively. follows

In the present study, physical quantities, skin friction coefficient and Nusselt number are defined as

$$
C_{f}=\frac{\tau_{w}}{\rho w_{e}^{2}(z) / 2}, \quad \mathrm{Nu}=\frac{a q_{w}}{\kappa\left(T_{w}-T_{\infty}\right)}
$$

where $\tau_{w}$ and $q_{w}$ shear stress and heat transfer rate from the surface of the cylinder, respectively, which given as

$$
\begin{aligned}
& \tau_{w}=\mu\left(\frac{\partial w}{\partial r}\right)_{r=a}, \quad q_{w}=\left[-\kappa\left(\frac{\partial T}{\partial r}\right)+q_{r}\right]_{r=a}, \\
& \tau_{w}=\frac{4 \mu c}{a} z f^{\prime \prime}(1), \quad q_{w}=-\frac{\kappa\left(T_{w}-T_{\infty}\right)}{a}\left[1+\frac{4 N}{3}\right] 2 \theta^{\prime}(1) .
\end{aligned}
$$

Substituting Eq.(4.6) in Eq.(4.5), we get

$$
C_{f}\left(\frac{z \mathrm{Re}}{a}\right)=f^{\prime \prime}(1), \quad \mathrm{Nu}=-2\left(1+\frac{4 N}{3}\right) \theta^{\prime}(1) .
$$




\section{Numerical solution}

The system of nonlinear ordinary differential Eqs (2.6) and (3.5) with the boundary conditions (2.7) and (3.6) respectively, is solved numerically using the fourth-order Runge Kutta scheme with the shooting technique. In this technique, first we convert the BVP into IVP (initial value problem). We set the following first-order systems

$$
\begin{aligned}
& f^{\prime}=p, \quad p^{\prime}=q, \quad q^{\prime}=\left[\left(M^{2}+\frac{1}{K}\right) p-\operatorname{Re}\left(f q-p^{2}\right)-q\right] / \eta \\
& \theta^{\prime}=z, \quad z^{\prime}=\left[-\left(1+\operatorname{Re} \operatorname{Pr} f+\frac{2 N}{3}\right) z-\frac{\operatorname{Re} \operatorname{Pr} \lambda}{2} \theta-\operatorname{Ec} \operatorname{Pr} q^{2} \eta\right] / \eta\left(1+\frac{4 N}{3}\right),
\end{aligned}
$$

with the boundary conditions

$$
f(1)=0, \quad p(l)=1+\alpha q(l), \quad \theta(1)=1+\beta z(l) .
$$

For solving the IVP, set of Eqs (4.5) and (4.6), we need the initial guesses for $q(1)=f^{\prime \prime}(l)$ and $z(l)=\theta^{\prime}(l)$ which are arbitrarily chosen and for adjusting the estimated value of $f^{\prime \prime}(1)$ and $\theta^{\prime}(l)$ we compare the calculated values for $f^{\prime}$ and $\theta$ at $\eta=10$ (say) with the given boundary conditions $f^{\prime}(10)=0, \theta(10)=0$ and applying the fourth-order Runge-Kutta method, a solution was obtained. The step-size is taken as $\Delta \eta=0.01$. This process was repeated until we obtained correct results up to the desired accuracy of $10^{-6}$ level.

\section{Result and discussion}

In the present study, a representative set of numerical values to all embedded parameters are assigned in order to analyze the velocity profile, temperature distribution, entropy generation and Bejan number with the help of Figs 2-12. The validation of accuracy of the applied numerical result is plotted in Fig. $4 \mathrm{~b}$ by assuming $\mathrm{Ec}=0$ (absence of viscous dissipation), $N=0$ (without radiation) and $\lambda=0$ (absence of heat source) for a fixed value of Pr. The present numerical result is found to be in good agreement with the available result Chauhan et al. [14].

Figure 2 shows the effect of pertinent parameters on the velocity profile. It is noted that velocity attains its maximum value at the surface of the cylinder and decreases as it moves away from the stretched surface of the cylinder. Velocity decreases as the magnetic field parameter $(M)$, slip parameter $(\alpha)$ and Reynolds number $(\mathrm{Re})$ increase whereas velocity increases as permeability increases. It is due to the reason that Darcy's resistance force decreases as permeability increases, therefore the flow increases. Also the applied transverse magnetic field rises the Lorentz force in the fluid flow regime which reduces the flow velocity and due to slip, the flow velocity near the stretching wall is no longer equal to the stretching velocity of the wall hence, the pulling of the stretching wall can only be partly transmitted to the fluid. 


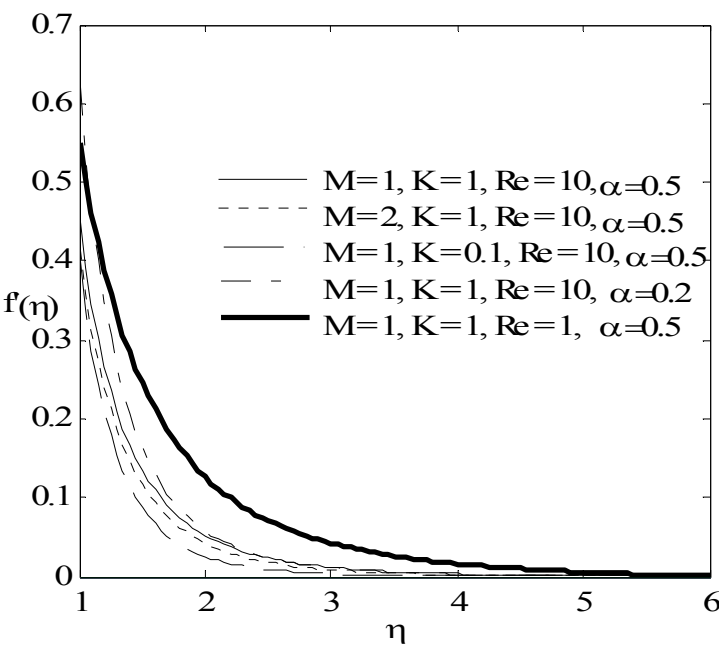

Fig.2. Velocity profile.

Figures 3-5 depict the temperature profiles for various values of the different parameters in the presence of the heat generating source. Temperature decreases as the distance from the surface of the stretching cylinder increases, and finally vanishes at some large distance from the stretching surface of the cylinder. The influence of the velocity slip $\alpha$, temperature slip parameter $\beta$, local Eckert number Ec and radiation parameter $N$ on the temperature distribution is plotted in Figs 3a-3b. The local Eckert number is defined as the ratio of kinetic energy at the wall to the specific enthalpy difference between the wall and fluid. As heat energy is stored in the fluid due to the frictional heating, temperature rises when the Ec number increases. Temperature increases with an increase in the velocity slip as shown in Fig.3a. While in Fig.3b noticed reverse effect on an increment of temperature slip, because heat generation rate is reduced with increasing effect of temperature slip parameter. Also we observed that temperature rises when radiation effect enhance, implies contribution of thermal radiation transfer in fluid flow increases which generates more heat flux and due this reason temperature increases with increasing value of $N$.

a)

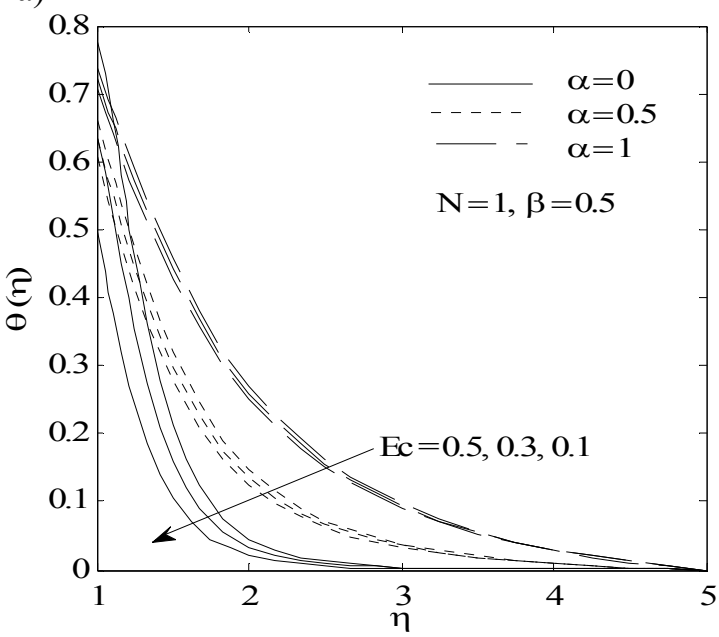

b)

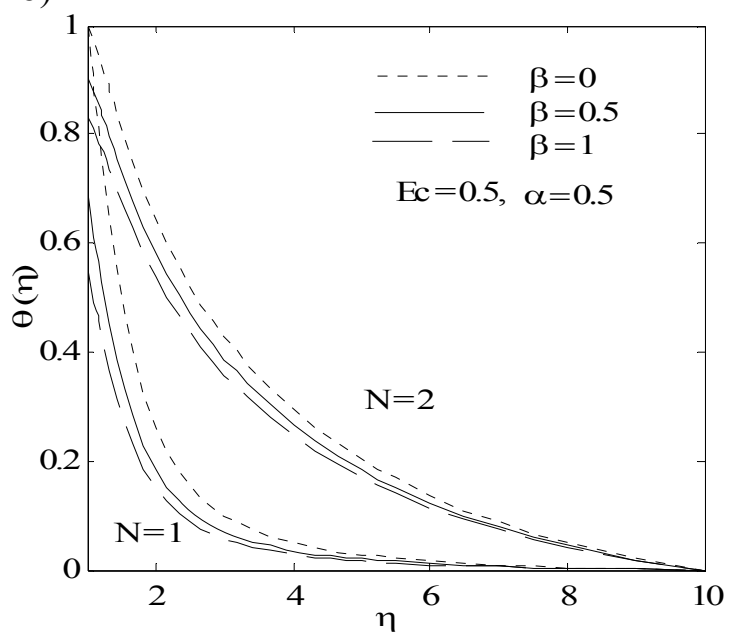

Fig.3. Effect of variation in (a) Eckert number and velocity slip parameter (b) Radiation parameter and temperature slip parameter on the temperature profile when $K=1, M=1, \operatorname{Pr}=5, \operatorname{Re}=10, \lambda=0.1$. 
Figures 4a-b illustrates the effect of the magnetic field parameter $M$, Reynolds number Re, Prandtl number $\operatorname{Pr}$ and permeability parameter $K$ on temperature profile. It is noticed that from Fig.4a that temperature rises when the magnetic field the increases due to the fact that the introduction of a transverse magnetic field tends to give a rise to the Lorentz force, to slows down the motion of the fluid flow and increases the thermal boundary layer. It is noted that temperature decreases when Re increases due to the fact that at low Reynolds numbers viscous force dominates but as the value of Re increases, viscous force decreases and temperature falls down. Figure $4 \mathrm{~b}$ noticed that temperature decreases with the increasing value of $K$ due to decreasing Darcy's resistance force. An increment in Pr causes a decrease in the thermal boundary layer thickness because of physical nature of Pr. An increasing value of Pr indicates an increasing viscous effect which implies that the viscous boundary layer is thicker than the thermal boundary, so a large value of Pr results in thinning of the thermal boundary layer.

Figures $5 \mathrm{a}-\mathrm{b}$ illustrates the influence of the heat generation/absorption parameter $(\lambda)$ on the temperature profile. The source term represents the heat generation distributed everywhere when $\lambda$ is positive. The heat absorption takes place in the case when $\lambda$ is negative and when $\lambda$ is zero is the case of no heat source. We observed both for slip condition and without slip condition. An increment in the value of the heat generation coefficient $\lambda$ causes the fluid temperature to increase. An increase in heat generation accelerates the flow, therefore the presence of progressively stronger heat source is beneficial to the regime. It is observed that for large heat generation effects $(\lambda=0.5)$ a distinctive peak in the temperature profile occurs in the region close with no slip conditions. A similar result has been obtained by Chamkha [23].

a)

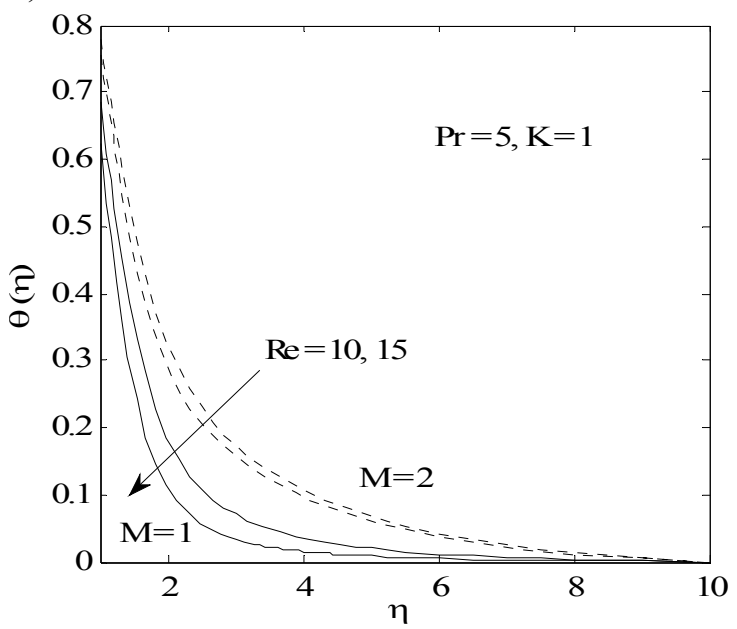

b)

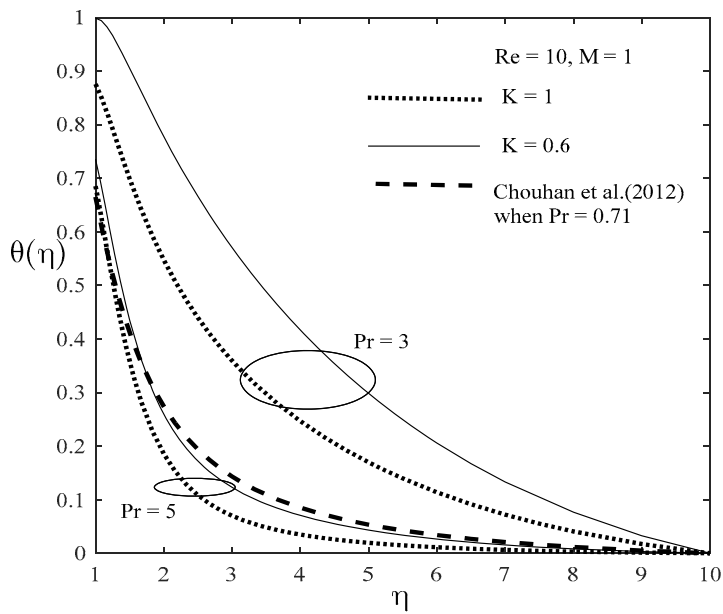

Fig.4. Effect of variation in (a) Reynolds number and magnetic parameter (b) permeability parameter and Prandtl number on the temperature profile when $\alpha=\beta=0.5, N=1, \mathrm{Ec}=0.5, \lambda=0.1$.

Table 1 presents the effect of various parameter magnetic parameter $M$, permeability parameter $K$, heat generation/absorption parameter $\lambda$, Reynolds number Re, Prandtl number Pr and radiation parameter $N$ on the numerical value of $f^{\prime \prime}(l)$ and $\theta^{\prime}(l)$ at constant slip condition. We observed that $f^{\prime \prime}(l)$ increases as $M$, Re and $\lambda$ increase and reduces when $\mathrm{K}, \operatorname{Pr}$ and $\mathrm{N}$ increase while $\theta^{\prime}(l)$ increases with an increment of $K$, $\operatorname{Pr}$ and Re and decrease for the rest of the parameters. Physically, a negative sign of $f^{\prime \prime}(1)$ implies that the surface exerts a dragging force on the fluid and a positive sign implies the opposite. 
a)

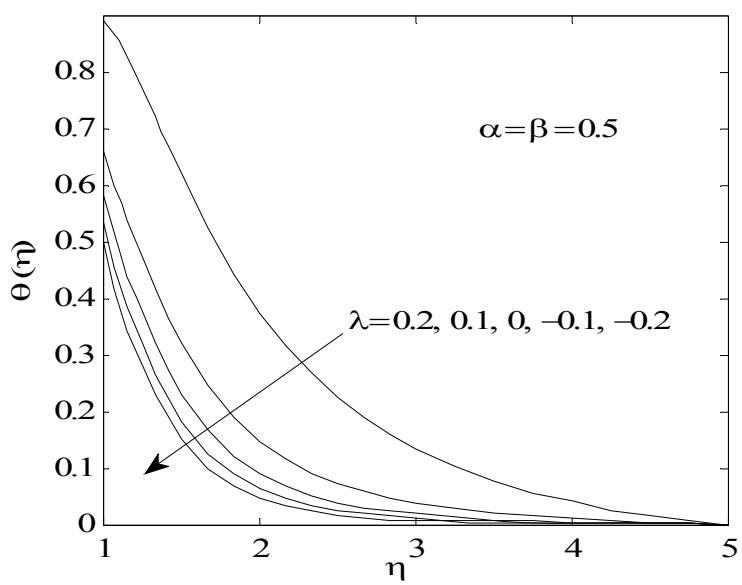

b)

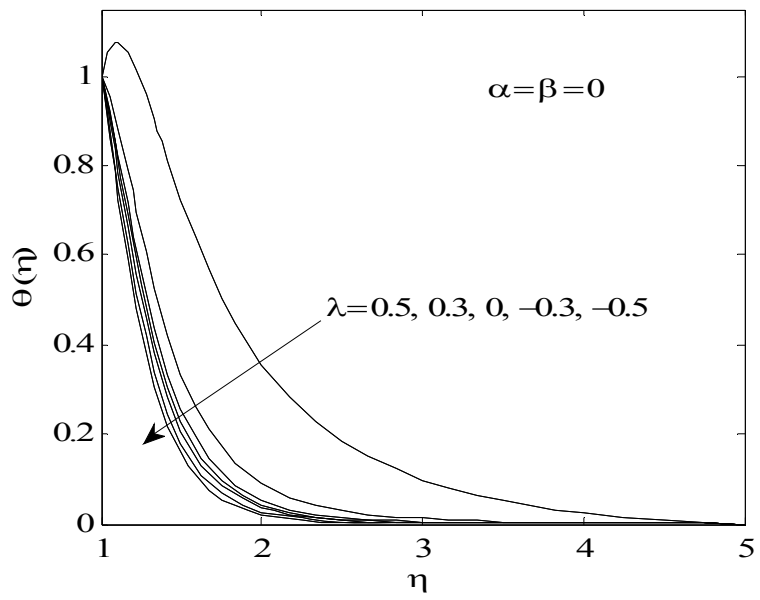

Fig.5. Effect of variation in (a) heat generation/absorption parameter with slip (b) heat generation/absorption parameter without slip, when $a=b=0.5, N=1, \mathrm{Ec}=0.5, K=1, M=1, \operatorname{Pr}=5, \operatorname{Re}=10$.

The effect of various parameters on the entropy generation number has been plotted in Figs 6-8. Figure 6a depicts that entropy enhances near the surface of the stretching cylinder with an increment in the Reynolds number $(\mathrm{Re})$ and after a critical point it reduces due to heat source, while the radiation effect $(N)$ enhances entropy throughout the cylinder because the heat transfer rate increases as the radiation parameter increases. In Fig.6b we can see that by increasing the value of the magnetic parameter $(M)$ entropy enhances near the surface of the stretching cylinder due to increases in the magnetic field parameter, Lorentz force rises which opposes the fluid motion as a result entropy attains a maximum value at the stretching surface of the cylinder but as this distances increasing entropy decreases and at far away it vanishes. It has been shown in Fig.6b that the result obtained in the present investigation are similar to those obtained by Butt and Ali (2014) which shows validation of our observations.

Table 1. Variation of numerical value of $f^{\prime \prime}(l)$ and $\theta^{\prime}(1)$ with $\lambda, K, M, \operatorname{Re}, \operatorname{Pr}$ and $N$ parameters, when $\alpha=\beta=0.5$ and $\mathrm{Ec}=0.2$.

\begin{tabular}{cccccccc}
\hline $\boldsymbol{M}$ & $\boldsymbol{K}$ & $\boldsymbol{N}$ & $\mathbf{P r}$ & $\mathbf{R e}$ & $\lambda$ & $f^{\prime \prime}(1)$ & $\theta^{\prime}(1)$ \\
\hline 1 & 1 & 1 & 5 & 10 & 0.1 & -1.150137893 & -0.718225801 \\
2 & 1 & 1 & 5 & 10 & 0.1 & -1.179971068 & -0.551196684 \\
1 & 1 & 1 & 5 & 10 & 0.1 & -1.150137893 & -0.718225801 \\
1 & 10 & 1 & 5 & 10 & 0.1 & -1.119716259 & -0.792298225 \\
1 & 1 & 1 & 5 & 10 & 0.1 & -1.150137893 & -0.718225801 \\
1 & 1 & 2 & 5 & 10 & 0.1 & -1.150137535 & -0.283862547 \\
1 & 1 & 1 & 5 & 10 & 0.1 & -1.150137893 & -0.718225801 \\
1 & 1 & 1 & 10 & 10 & 0.1 & -1.150137275 & -1.006527446 \\
1 & 1 & 1 & 5 & 10 & 0.1 & -1.150137893 & -0.718225801 \\
1 & 1 & 1 & 5 & 15 & 0.1 & -1.204112472 & -0.831485523 \\
1 & 1 & 1 & 5 & 10 & -0.1 & -1.150138655 & -0.992486288 \\
1 & 1 & 1 & 5 & 10 & 0 & -1.150138659 & -0.902881000 \\
1 & 1 & 1 & 5 & 10 & 0.1 & -1.150137893 & -0.718225801 \\
\hline
\end{tabular}


a)

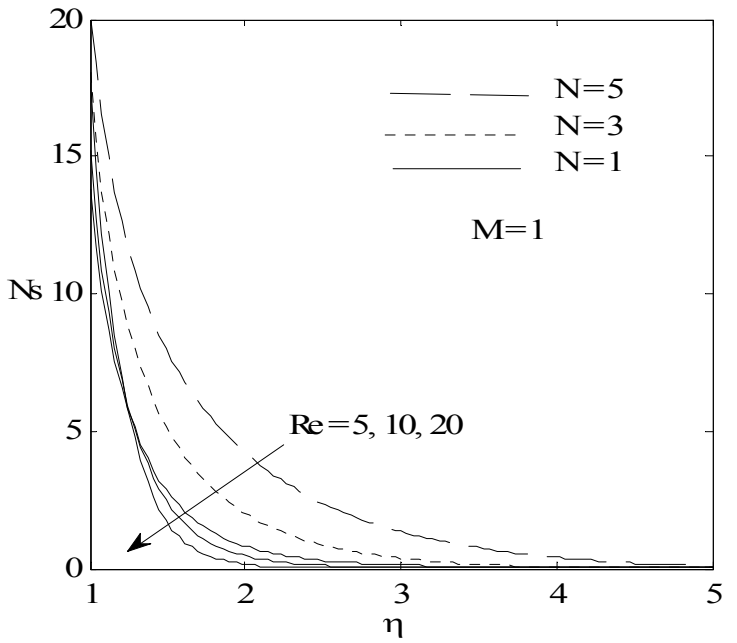

b)

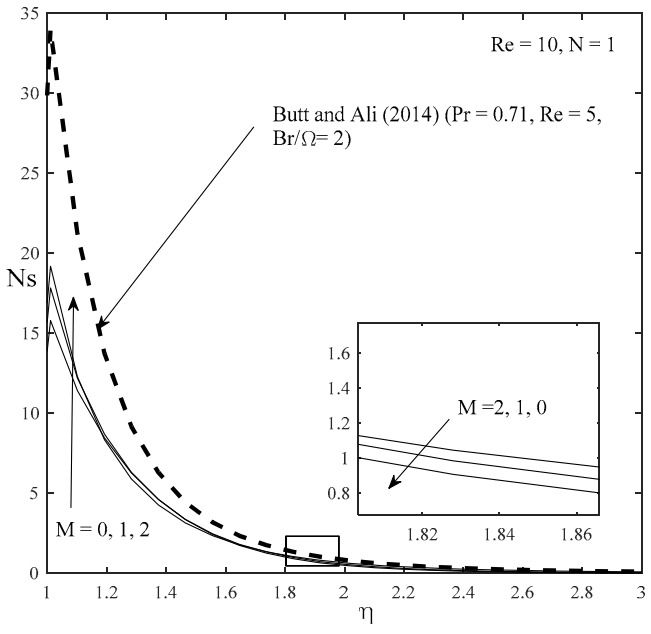

Fig.6. Effect of variation in (a) Reynolds number and radiation parameter (b) magnetic parameter on the Entropy generation number when $\alpha=\beta=0.5, K=1, \operatorname{Pr}=5, \mathrm{Ec}=0.5, \lambda=0.1, \mathrm{Br} / \Omega=5, X=0.5$.

Figure 7a shows that the entropy generation number $(N s)$ decreases as velocity slip parameters $(\alpha)$ increases. Figure $7 \mathrm{~b}$ shows that entropy generation increases near the surface of the stretching cylinder as the Prandtl number (Pr) increases, it is due to decreases in the temperature gradient but after certain thickness of the boundary layer it starts to decrease due to the presence of heat source. The effects of the group parameter $(\mathrm{Br} / \Omega)$ and Eckert number $(\mathrm{Ec})$ on $N s$ are depicted in Fig.8a. Increasing value of heat generation or absorption parameter $(\lambda)$, also increases the entropy generation number $(N s)$ as shown in Fig.8b.

a)

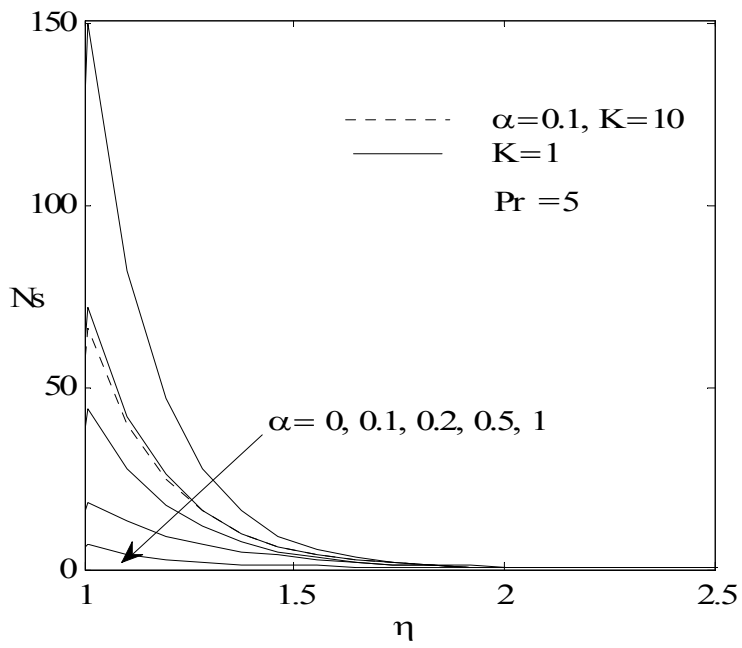

b)

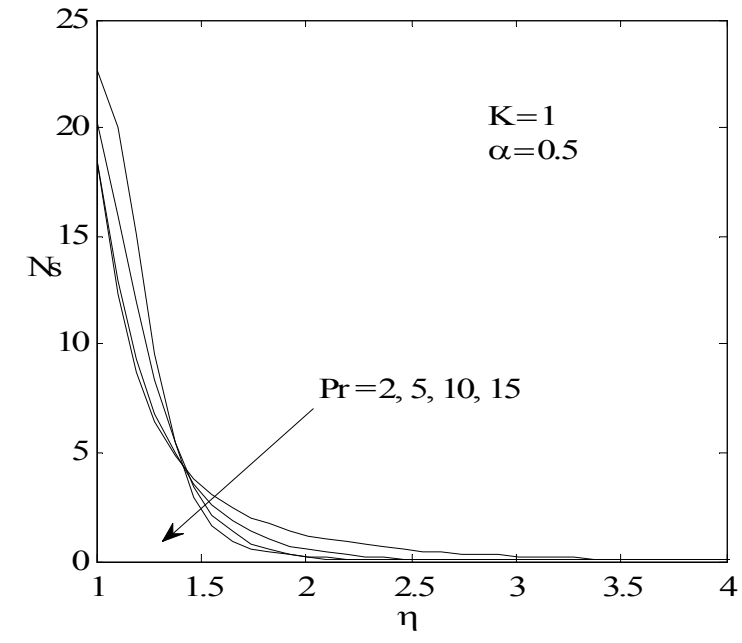

Fig.7. Effect of variation in (a) velocity and permeability parameters (b) Prandtl number on the entropy generation number when $M=1, N=1, \operatorname{Re}=10, \mathrm{Ec}=0.2, \lambda=0.1, \mathrm{Br} / \Omega=5, X=0.5$. 
a)

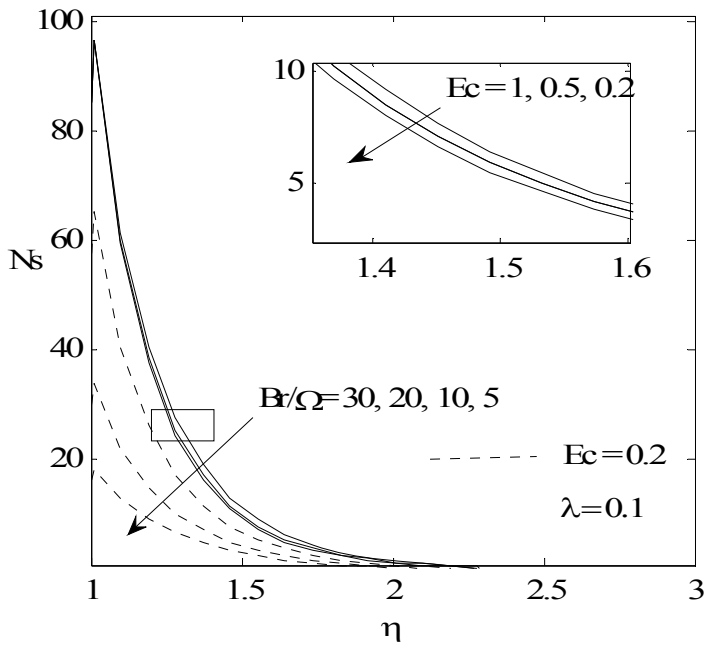

b)

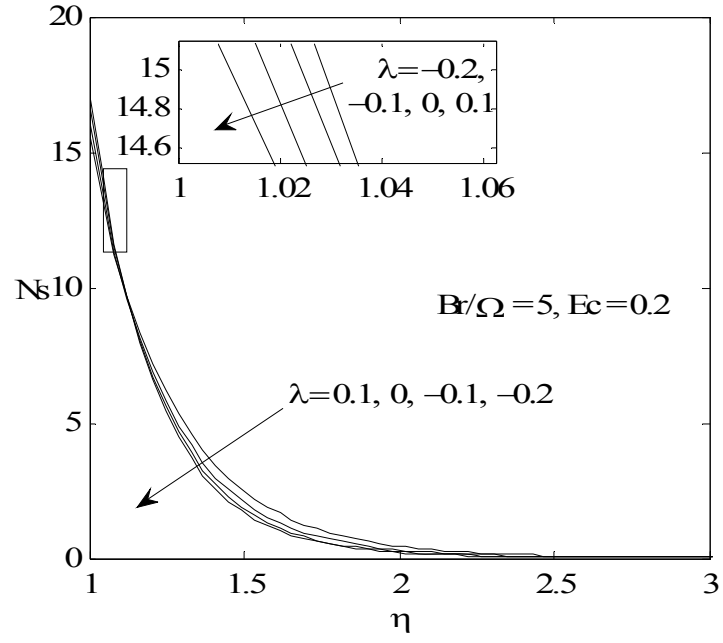

Fig.8. Effect of variation in (a) group parameter and Eckert number (b) heat generation/absorption parameter on the entropy generation number when $M=1, K=0.5, N=1, \operatorname{Re}=10, \alpha=\beta=0.5, \operatorname{Pr}=5, X=0.5$.

Figures 9-10 illustrate the effects of various parameters on the Bejan number $(\mathrm{Be})$. It is shown that Be increases with the increasing value of the Reynolds number $(\mathrm{Re})$, magnetic field parameter $(M)$ and Eckert number $(\mathrm{Ec})$ while shows reverse effect for increasing group parameter $(\mathrm{Br} / \Omega)$ in stretching cylinder which is shown in Figs 9a-b and in Figs 10a-b, we observed that Be decreases as the temperature slip parameter $(\beta)$ and heat absorption/generation parameter $\lambda$ increase. The value of Be reduces to the surface of the stretching cylinder but after a point of inflexion it shows little increment with the increasing radiation effect and Fig.10b illustrates that the velocity slip parameter $(\alpha)$ enhances the Bejan number.

a)

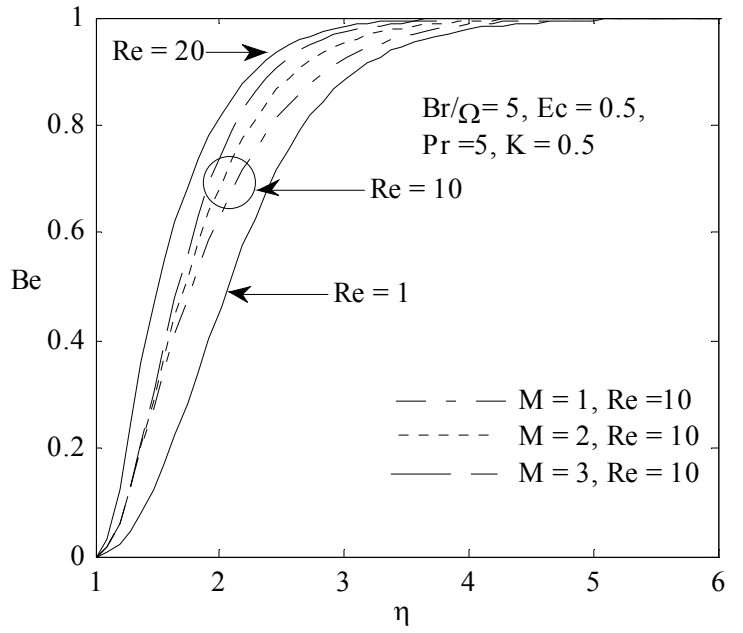

b)

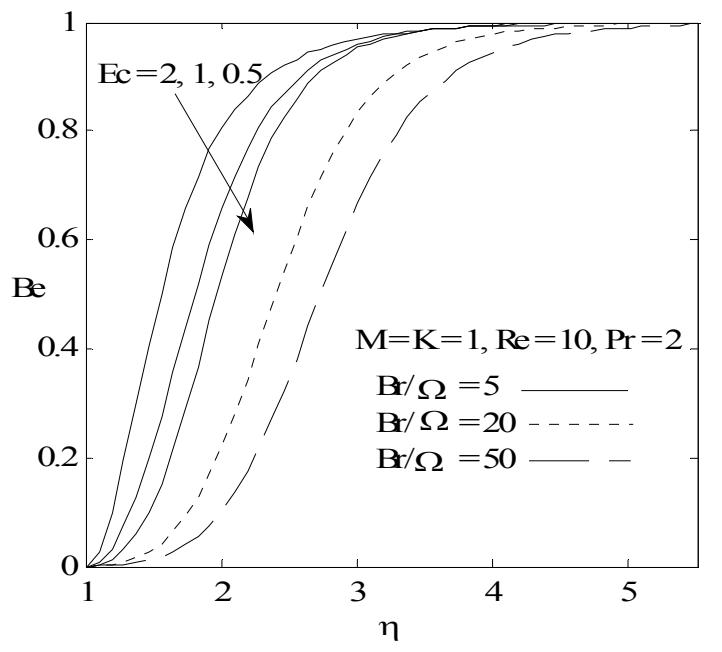

Fig.9. Effect of variation in (a) Reynolds number and magnetic parameter (b) group parameter and Eckert number on the Bejan number when $\alpha=\beta=0.5, \lambda=0.1, X=0.5$. 
a)

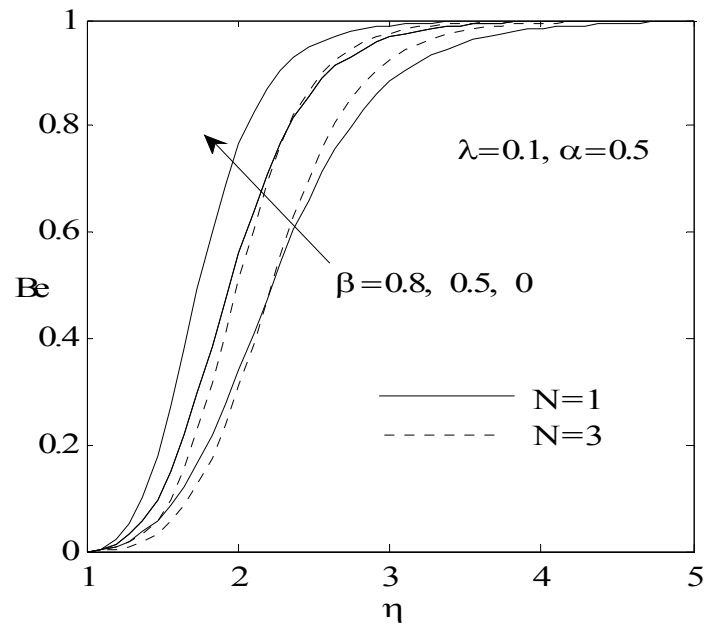

b)

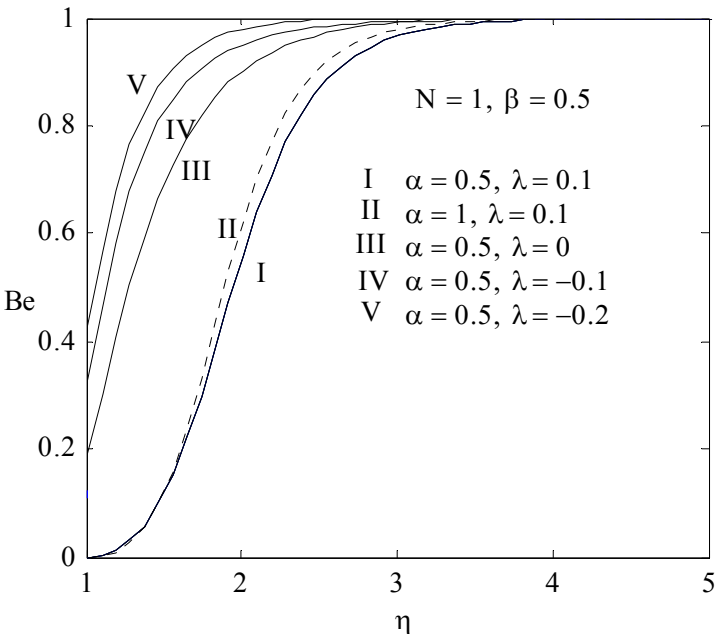

Fig.10. Effect of variation in (a) temperature slip parameter and radiation parameter (b) heat generation/absorption parameter and velocity slip parameter on the Bejan number when $\mathrm{Ec}=0.5$, $\mathrm{Re}=10, M=1, K=0.5, \operatorname{Pr}=2, \mathrm{Br} / \Omega=5, X=0.5$.

\section{Conclusion}

The following conclusions are drawn from the investigation:

- The magnetic field causes resistance to the flow which results in a decrease in velocity and increase in temperature.

- The thermal boundary layer thickness decreases with the Prandtl number Pr, temperature slip parameter $\beta$ and increases with Eckert number Ec, velocity parameter $\alpha$ and radiation parameter.

- The entropy generation number $N S$ increases near the surface after a point it reduces with magnetic parameter $M$, Reynolds number Re and Prandtl number Pr.

- The ratio of entropy due to heat transfer and total entropy increases as velocity slip increases and shows reverse effect as temperature slip increases.

\section{Conflict of interest}

The author's declares there is no conflict of interest in publication of this article.

\section{Nomenclature}

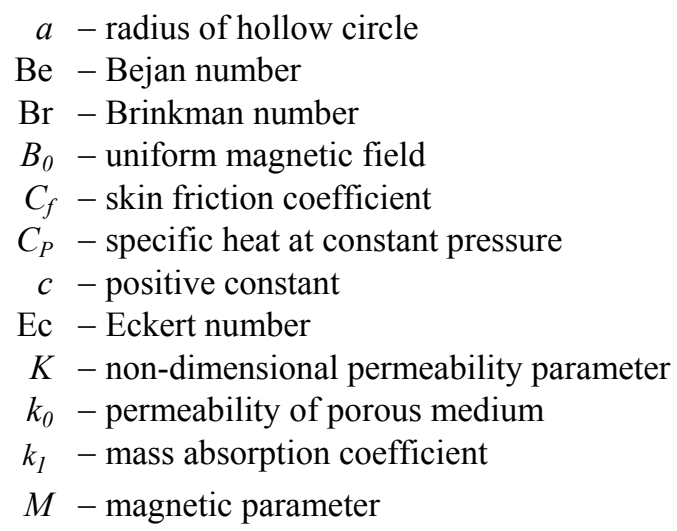




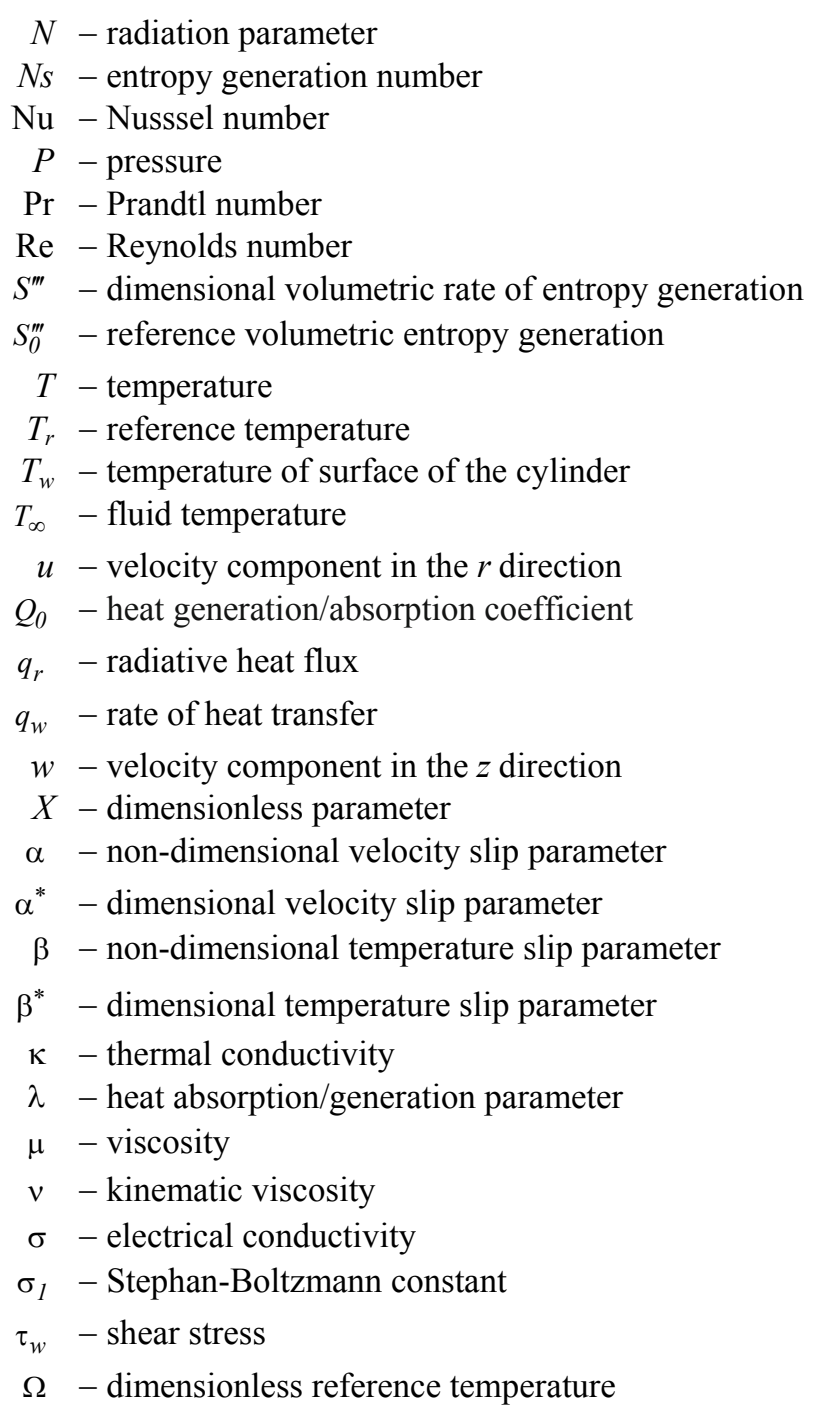

\section{References}

[1] Lin H.T. and Shih Y.P. (1980): Laminar boundary layer heat transfer along static and moving cylinders. - J. Chin. Inst. Eng., vol.3, pp.73-79.

[2] Lin H.T. and Shih Y.P. (1981): Buoyancy effects on the laminar boundary layer heat transfer along vertically moving cylinders. - J. Chin. Inst. Eng., vol.4, pp.47-51.

[3] Wang C.Y. (1988): Fluid flow due to a stretching cylinder. - Phys. Fluids, vol.31, pp.466-468.

[4] Burde HI. (1989): On the motion of fluid near stretching circular cylinder. - PMM USSR vol.53, pp.271-273.

[5] Ishak A. and Nazar R. (2009): Laminar boundary layer flow along a stretching cylinder. - Eur. J. Sci. Res., vol.36, pp.22-29.

[6] Bachok N. and Ishak A. (2010): Flow and heat transfer over a stretching cylinder with prescribed surface heat flux. - Malaysian J. Math. Sci., vol.4, pp.159-169.

[7] Sparrow E.M. and Gregg J.L. (1956): Laminar free convection heat transfer from the outer surface of a vertical circular cylinder. - ASME J. Heat Transfer, vol.78, pp.1823-1829. 
[8] Chamkha A.J. and Quadri M.M.A. (2001): Heat and mass transfer from a permeable cylinder in a porous medium with magnetic field and heat generation=absorption effects. - Numer. Heat Transfer, Part A., vol.40, pp.387-401.

[9] Saeid N.H. (2006): Analysis of free convection about a horizontal cylinder in a porous media using a thermal non-equilibrium model. - Int. Commu. Heat MassTrans., vol.33, pp.158-165.

[10] Nguyen H.D., Paik S. and Douglass R.W. (1996): Unsteady mixed convection about a rotating circular cylinder with small fluctuations in the free-stream velocity. - Int. J. Heat Mass Transfer, vol.39, pp.511-525.

[11] Gang F.T., Ji Z., Fang Z.Y. and Hua T. (2008): Unsteady viscous flow over an expanding stretching cylinder. - Chin. Phys. Lett., vol.124, No.28, pp.707-710.

[12] Wang C.Y. and Ng C.O. (2011): Slip flow due to a stretching cylinder. - Int. J. Non-Linear Mechanics, vol.46, pp.1191-1194.

[13] Mukhopadhyay S. (2011): Chemically reactive solute transfer in a boundary layer slip flow along a stretching cylinder. - Int. J. Chem. Sci. Eng, vol.5, pp.385-391.

[14] Chauhan D.S., Rastogi P. and Agrawal R. (2012): Magnetohydrodynamic slip flow and heat transfer in a porous medium along a stretching cylinder: Homotopy Analysis Method. Numerical heat transfer, Part A: Application: Int. J. Comp. Method., vol.62, No.2, pp.136-157.

[15] Chauhan D.S., Rastogi P. and Agrawal R. (2014): Magnetohydrodynamic flow and heat transfer in a porous medium along a stretching cylinder with radiation: Homotopy Analysis Method. - Afrika Matematika, vol.25, No.1, pp.115-134.

[16] Jain S. and Parmar A. (2017): Radiation effect on MHD williamson fluid flow over stretching cylinder through porous medium with heat source. - Lect. Notes Mech. Engg., Springer, pp.61-78.

[17] Jain S. and Bohra S. (2016): Radiation effects in flow through porous medium over a rotating disk with variable fluid properties. - Adv. Math. Phys., vol.2016, pp.1-12.

[18] Jain S. and Bohra S. (2017): Hall current and radiation effects on unsteady MHD squeezing nanofluid flow in a rotating channel with lower stretching permeable wall. - Lect. Notes Mech. Engg., Springer, pp.127-141.

[19] Abbas Z., Majeed A. and Javed T. (2013): Thermal radiation effects on MHD flow over a stretching cylinder in a porous medium. - Heat Transf. Res., vol.44, No.8, pp.703-718.

[20] Jain S. and Chaudhary R. (2017): Combined effects of sunction/injection on MHD boundary Layer flow of nanofluid over horizontal permeable cylinder with radiation. - J. Advanced Research in Dynamical and Control System, vol.11, pp.88-98.

[21] Jain S. and Chaudhary R. (2017): Soret and Dufour effects on MHD fluid flow due to moving permeable cylinder with radiation. - Globaland Stochastic Analysis, pp.75-84.

[22] Abel M.S., Nandeppanavar M.M. and Malkhed M.B. (2010): Hydromagnetic boundary layer flow and heat transfer in viscoelastic fluid over a continuously moving permeable stretching surface with non-uniform heat source/sink embedded in fluid-saturated porous medium. - Chem. Eng. Commun., vol.197, pp.633-655.

[23] Chamkha A.J. (2011): Heat and mass transfer from MHD flow over a moving permeable cylinder with heatgeneration or absorption and chemical reaction. - Commu. Numerical Analysis, Article ID cna-00109, 20 pages doi: 10.5899/2011/cna-00109.

[24] Manjunatha P.T., Gireesha B.J. and·Prasannakumara B.C. (2015):Effect of radiation on flow and heat transfer of MHD dusty fluid over a stretching cylinder embedded in a porous medium in presence of heat source. - Int. J. Appl. Comput. Math, vol.2015, pp.1-18.

[25] Bejan A. (1982): Entropy generation through heat fluid flow. - 2nd ed. New York: Wiley.

[26] Makinde O.D. and Beg O.A. (2010): On inherent irreversibility in a reactive hydromagnetic channel flow. - J. Thermal Sci., vol.19, pp.72-79. 
[27] Jain S., Kumar V. and Bohra S. (2015): Entropygeneration in pressure gradient assisted Couette flow in porous medium with different thermal boundary conditions. -Int. J. Energy and Tech., vol.7, pp.40-48.

[28] Jain S., Kumar V. and Bohra S. (2017): Entropy generation for MHD radiative compressible fluid flow in a channel partially filled with porous medium. - Global and Stochastic Analysis, pp.13-31.

[29] Bak A.H. and Heilen W.N. (1999): Entropy generation due to laminar natural convection over a heated rotating cylinder. - Int. J. Heat Mass Transfer, vol.42, pp.4225-4233.

[30] Yilbas B.S. (2001): Entropy analysis of concentric annuli with rotating outer cylinder.- Int. J. Energy, vol.1, pp.60-66.

[31] Butt A.S. and Ali A. (2013): Effects of magnetic field on entropy generation in flow and heat transfer due to radially stretching surface. - Chin. Phys. Lett., vol.30, pp.024701- 24704.

[32] Munawar S., Mehmood A. and Ali A. (2012):Thermal analysis of the flow over an oscillatory stretching cylinder. - Phys. Scr., vol.86, pp.65401-65412.

[33] Butt A.S. and Ali A. (2014):Entropy analysis of magnetohydrodynamic flow and heat transfer due to a stretching cylinder. - J. Taiwan Inst. Chemical Engineers, vol.45, pp.780-786.

[34] Paoletti S., Rispoli F. and Sciubba E. (1989): Calculation of exegetic losses in compact heat exchanger passages. - ASME AES, vol.10, pp.21-29.

Received: February 1, 2017

Revised: December 28, 2017 